\title{
Pion production in nucleon-nucleon collisions near threshold: complete NNLO calculation in chiral EFT
}

\author{
A. A. Filin ${ }^{* a}$, V. Baru ${ }^{a b}$, E. Epelbaum ${ }^{a}$, C. Hanhart ${ }^{c}$, H. Krebs ${ }^{a}$, and F. Myhrer ${ }^{d}$ \\ ${ }^{a}$ Institut für Theoretische Physik II, Ruhr-Universität Bochum, D-44780 Bochum, Germany \\ ${ }^{b}$ Institute for Theoretical and Experimental Physics, 117218, B. Cheremushkinskaya 25, Moscow, \\ Russia \\ ${ }^{c}$ Institut für Kernphysik, (Theorie) and Jülich Center for Hadron Physics, Forschungszentrum \\ Jülich, D-52425 Jülich, Germany and Institute for Advanced Simulation, Forschungszentrum \\ Jülich, D-52425 Jülich, Germany \\ ${ }^{d}$ Department of Physics and Astronomy, University of South Carolina, Columbia, SC 29208, USA \\ E-mail: arseniy.filin@tp2.rub.de
}

\begin{abstract}
The reaction $N N \rightarrow N N \pi$ is a useful tool to test chiral effective field theory at intermediate energies, understand mechanisms of the first inelastic channel in NN interactions and study charge symmetry breaking. In order to make high accuracy predictions and due to various cancellations at leading and next-to-leading orders, it is important to consider higher order terms in the chiral expansion. We present the results for the pion production operator near threshold calculated up-to-and-including next-to-next-to-leading order in chiral effective field theory, which includes pions, nucleons and delta degrees of freedom. We calculate observables for the $p p \rightarrow d \pi^{+}$channel by performing the convolution of the obtained operators with nucleon-nucleon wave functions based on modern phenomenological and chiral potentials.
\end{abstract}

The 8th International Workshop on Chiral Dynamics

29 June 2015 - 03 July 2015

Pisa, Italy

\footnotetext{
* Speaker.
} 


\section{Introduction}

Study of near-threshold pion production is important to understand mechanisms of the first inelastic channel in nucleon-nucleon (NN) scattering, test chiral effective field theory (EFT) at intermediate energies, and get information about charge symmetry breaking (CSB) in few-nucleon processes (see refs. [1,2] for extensive review articles). Pion production from two nucleons contributes to many few-nucleon processes as a building block, can be investigated directly in the inelastic nucleon-nucleon reactions, such as $p p \rightarrow d \pi^{+}$and $p p \rightarrow p p \pi^{0}$ and provides access to the CSB observables in $p n \rightarrow d \pi^{0}$.

Neutral pion production in the $p p \rightarrow p p \pi^{0}$ channel has been known to be the most puzzling process since the pioneering work of Koltun and Reitan [3], see also Refs. [4, 5, 6, 7, 8, 9] for more recent studies within chiral EFT. ${ }^{1}$ In this channel the measured near-threshold cross section is suppressed by more than an order of magnitude compared to charged pion-production channels. This suppression is in line with conclusions made using chiral EFT, namely that there is almost no leading order contribution to $p p \rightarrow p p \pi^{0}$ [4] and that higher order effects are expected to be important for a quantitative understanding of neutral pion production $[4,8,9]$.

Pion production in $p n \rightarrow d \pi^{0}$ channel is important in order to study CSB in strong interactions [10]. Specifically, the experimentally measured differential-cross-section asymmetry [11] in this channel can be used to extract the strong part of the neutron-proton mass difference $[12,13,14]$. In order to reliably extract CSB observables, it is imperative to have an accurate calculation of isospin-symmetric observables and to ensure that the chiral expansion converges. For this purpose the $p p \rightarrow d \pi^{+}$channel is a good starting point since: (i) unlike $p p \rightarrow p p \pi^{0}$ no suppressions of leading-order (LO) contribution takes place in this channel $[3,15,8,9]$ and (ii) precise experimental data from hadronic atom measurements are available for comparison [16, 17].

In this work we follow the lines of Ref. [18] to discuss the pion production operator calculated up to next-to-next-to-leading order (NNLO) in Refs. [8, 9] and test the convergence of chiral expansion of observables in $p p \rightarrow d \pi^{+}$.

This paper is organized as follows. In section 2 we define relations between observables and amplitudes in $p p \rightarrow d \pi^{+}$channel. In section 3 we discuss the methods used to calculate the amplitudes for $N N \rightarrow N N \pi$ processes in chiral EFT framework. In section 4 we present the complete NNLO operators for s-wave pion production. In Sections 5 and 6 we consider convolution of NNLO pion production operators with phenomenological and chiral NN wave functions, calculate observables and compare them with experimental data. We summarize our findings in section 7.

\section{Observables in $p p \rightarrow d \pi^{+}$channel}

The total near threshold cross section of $p p \rightarrow d \pi^{+}$reaction in center-of-mass system (CMS) can be parametrized as

$$
\sigma=\alpha \eta+\beta \eta^{3}
$$

\footnotetext{
${ }^{1}$ For an overview of the phenomenological approaches the interested reader is referred to the review articles [1, 2].
} 
where $\eta$ is the outgoing pion momentum in the units of pion mass. The differential cross section is expressed in terms of transition amplitude as:

$$
\frac{d \sigma}{d \Omega}=\frac{1}{64 \pi^{2}} \frac{\left|\vec{q}_{\pi}\right|}{\left|\vec{p}_{\text {in }}\right| \varepsilon_{\text {tot }}^{2}}|\bar{M}|^{2},
$$

where $\left|\vec{q}_{\pi}\right|=\eta m_{\pi}$ is outgoing pion momentum, $\vec{p}_{i n}$ is incoming proton momentum, $\varepsilon_{t o t}$ is the total energy in CMS, and $|\bar{M}|^{2}$ is the square of the transition amplitude averaged over the spins of the initial protons and summed over outgoing deuteron polarizations:

$$
|\bar{M}|^{2}=\frac{1}{2 s+1} \frac{1}{2 s+1} \sum_{\lambda_{1} \lambda_{2}} \sum_{\varepsilon_{d}}\left|M_{p p \rightarrow d \pi^{+}}\left(\lambda_{1}, \lambda_{2}, \vec{\varepsilon}_{d}, \vec{p}_{i n}, \vec{q}_{\pi}\right)\right|^{2},
$$

where $s=1 / 2$ is the total spin of the proton, $\lambda_{1}$ and $\lambda_{2}$ are projections of spin for each proton, and $\vec{\varepsilon}_{d}$ is the deuteron polarization vector. At threshold we can further simplify Eq. (2.2) using:

$$
\varepsilon_{t o t}=m_{d}+m_{\pi}, \quad\left|\vec{p}_{i n}\right|=\sqrt{\left(\frac{\varepsilon_{t o t}}{2}\right)^{2}-m_{N}^{2}}
$$

To include the effects of NN interaction in the initial and final state, it is convenient to perform a partial wave decomposition of the amplitude Eq. (2.3). At threshold the cross section becomes $\sigma=\alpha \eta$ and the only contribution to $\alpha$ comes from the initial $p p^{3} P_{1}$ partial wave ${ }^{2} M_{3 P 1}$

$$
\alpha=\frac{4 \pi}{64 \pi^{2}} \frac{m_{\pi}}{p_{i n}\left(m_{\pi}+m_{d}\right)^{2}} \frac{3}{4}\left|M_{3 P 1}\right|^{2} .
$$

The value of the parameter $\alpha$ at threshold can be extracted from the precise experiment on pionic deuterium lifetime performed at PSI [16, 17], where the total cross section for the channel $n n \rightarrow d \pi^{-}$was found to be $\sigma\left(n n \rightarrow d \pi^{-}\right)=252_{-11}^{+5} \eta \mu b$. Using these data and neglecting isospin breaking effects we can extract the absolute value of the amplitude $\left|M_{3 P 1}\right|$ at threshold:

$$
\left|M_{3 P 1}\right|^{\text {exp. }}=21.5_{-0.5}^{+0.2}
$$

This observable will be compared with our chiral-EFT-based prediction.

\section{Formalism}

Since pion-nucleon interactions at low energies are governed by the chiral symmetry properties of QCD, chiral effective field theory is a natural tool to study pion production reactions.

The transition amplitudes for the $N N \rightarrow N N \pi$ processes involve several ingredients, namely the pion production operator as well as NN interactions in the initial and final states. Since NN interactions are non-perturbative, they cannot be directly calculated in chiral perturbation theory. For this reason, the so-called hybrid approach [19] is used. In the hybrid approach, the full amplitude is calculated in two steps. As a first step, the irreducible pion production operator is calculated perturbatively in the chiral EFT framework. In the second step, this operator is convolved with NN wave

\footnotetext{
${ }^{2}$ To denote NN partial waves we use spectroscopic notation ${ }^{2 S+1} L_{J}$, where $S$ is a total spin, $L$ is orbital angular momentum and $J$ is the total angular momentum of $\mathrm{NN}$ pair.
} 
functions obtained from the solution of a non-perturbative Lippmann-Schwinger or Schrödinger equation with a realistic $\mathrm{NN}$ potential. It is important, that the production operator itself should not contain any parts of NN interaction in order to avoid double counting. For this reason, only irreducible production operators are considered, i.e. those which do not contain two-nucleon cuts. However, if the production operator involves energy-dependent vertices, one has to take special care in order to separate all irreducible contributions correctly, see Ref. [15] for the procedure of identifying irreducible contributions.

The hybrid approach can be generalized to include explicit delta degrees of freedom, see Refs. [20, 21] for the first steps in this direction and Refs. [22, 23, 24] for earlier phenomenological calculations. Explicit inclusion of delta degrees of freedom can be done using one of the following two strategies. One can either consider coupled channels where the pion-production operator is allowed to have external deltas (e.g. $N \Delta \rightarrow N N \pi$ ) and initial state interactions can have the form $N N \rightarrow N \Delta$ and $N N \rightarrow \Delta \Delta$. In this approach diagrams containing intermediate $N \Delta$ cuts are reducible. Alternatively, one only considers intermediate delta excitations in evaluating the pion production operator. In this latter case the production operator always has the form of $N N \rightarrow N N \pi$, and the NN interaction is limited to the elastic $N N \rightarrow N N$ channel. Since most of modern phenomenological and chiral NN potentials do not include $N N \rightarrow N \Delta$ channels explicitly, we use the latter strategy. In this formulation, all diagrams with intermediate $N \Delta$ cuts are irreducible by definition and should be calculated explicitly as parts of the production operator.

Technically, the convolution of the production operator with the initial and final NN wave functions is done by first performing a partial wave projection of the pion-production operator. The resultant operator is sandwiched between the $\mathrm{NN}$ wave functions and integrated up to a cutoff of order $600-1000 \mathrm{MeV}$. The equations defining the convolution procedure can be found in Appendix A of Ref. [9].

The pion production operator can be calculated perturbatively in the chiral EFT framework. However, it was shown in $[5,6,25,26,27,28]$ that the naive application of the standard power counting (where all typical momenta $p$ in the reaction are counted as of order of pion mass $m_{\pi}$ ) neither reproduces the data nor leads to convergent chiral expansion. It was suggested in refs. [4, 20] that the power counting should be modified to take into account the new small scale which is associated with typical transferred momenta $p=\sqrt{m_{\pi} m_{N}}$ in the pion-production processes $\left(m_{N}\right.$ is the nucleon mass). In such modified power counting, the expansion parameter is

$$
\chi_{\mathrm{MCS}} \simeq \frac{p}{\Lambda_{\chi}} \simeq \sqrt{\frac{m_{\pi}}{m_{N}}}
$$

where $\Lambda_{\chi} \simeq m_{N}$ corresponds to a typical hadronic scale. In what follows, we refer to this counting as momentum counting scheme (MCS). Furthermore, numerically $m_{\pi} / p$ appears to be equal to $\chi_{\text {MCs. }}$. Delta resonance can be also included in momentum counting scheme, assuming that the delta-nucleon mass difference is of order of $p$.

\section{S-wave pion-production operators}

We have calculated the expressions for pion production operators relevant for s-wave pion production up-to-and-including NNLO in the MCS. The complete expressions for operators are given in Ref [9]. In this section we summarize the most important results. 




Figure 1: Diagrams corresponding to s-wave pion-production operators up to NNLO. More NNLO diagrams with intermediate $\Delta$ are shown in Fig. 2. Dashed, solid, and double lines denote pions, nucleons and delta-resonances respectively. Notation of the vertices and corresponding Lagrangians are given in Ref. [9]. The red square in the box diagrams indicates that the corresponding nucleon propagator cancels with parts of the $\pi N$ vertex and leads to the irreducible contribution, see Ref. [9] for further details.

For LO operators only two types of terms contribute: the single-nucleon "direct" pion production operator, and the Weinberg-Tomozawa operator (including its recoil correction labelled WT recoil), see top row in Fig. 1.

At next-to-leading order (NLO), tree-level diagrams with intermediate delta excitations start to contribute (second row in Fig. 1). In addition, loop diagrams appear at NLO (third and fourth row in Fig. 1). However, as was pointed out in Refs. [7, 15], all NLO loop diagrams cancel out exactly.

Finally, at NNLO there are tree-level and loop diagrams, as well as five-point contact terms. In addition, there are numerous loop diagrams with explicit $\Delta$ (see Fig. 2). We have calculated all diagrams and found numerous cancellations among the loop diagrams at NNLO. Unlike NLO 


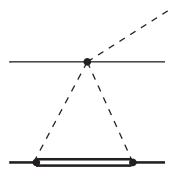

$\Delta \mathrm{II}$

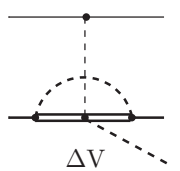

$\Delta \mathrm{V} \cdot$

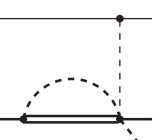

$\mathrm{VIb}$

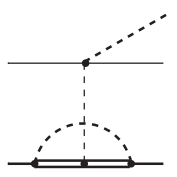

$\Delta \mathrm{X}$

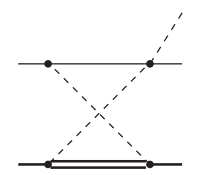

$\Delta \mathrm{III}$

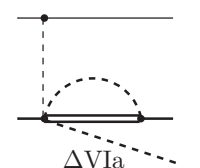

$\Delta \mathrm{VIàa} \cdot \cdots$

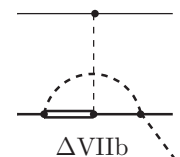

$\Delta \mathrm{VIIb} \because$

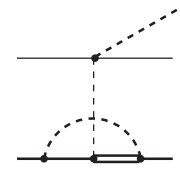

$\Delta \mathrm{XIa}$

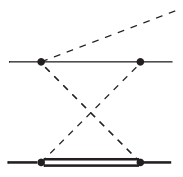

$\Delta \mathrm{IIIb}$

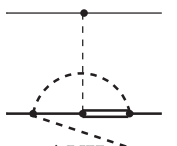

$\Delta$ viİà.

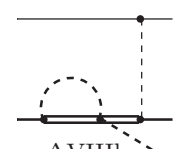

$\Delta$ VIIIb'

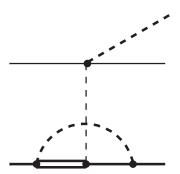

$\triangle \mathrm{XIb}$

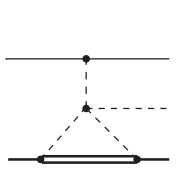

$\Delta \mathrm{IV}$

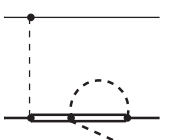

$\Delta$ viì̃à-
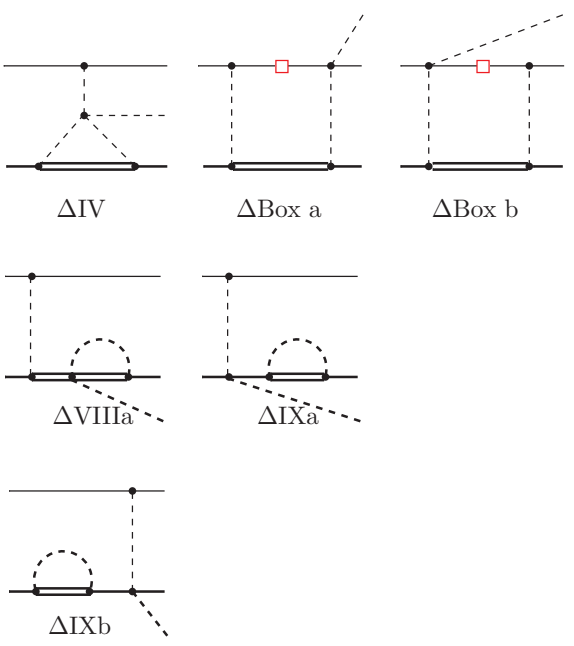

Figure 2: Loop diagrams with the delta degree of freedom contributing to the s-wave pion production at NNLO. Notation is the same as in Fig. 1.

loops, where cancellation is exact, a finite contribution remains after renormalization at NNLO. There is also a finite contribution from NNLO tree-level diagrams. The expressions for NNLO pion production operators are given in Ref. [9].

Next we consider the contributions of these operators to the $p p \rightarrow d \pi^{+}$observables.

\section{Results}

We have calculated the observables for $p p \rightarrow d \pi^{+}$by performing the convolution of pion production operators of section 4 (Figs. 1 and 2) with a set of NN wave functions derived from the modern phenomenological potentials: CDBonn [29], Nijmegen [30] and AV18 [31]. In what follows, the expression for the $N N \rightarrow N N \pi$ operator derived based on the diagrams shown in figures 1 and 2 will be called (s-wave) pion production operator while its convolution with the NN interaction in the initial and final state will be referred as the (s-wave) pion production amplitude. In this section we discuss the results of the convolution and compare the resulting amplitudes with the value extracted from experiment, see Eq. 2.6.

To test the convergence of chiral EFT, we find it instructive to consider contributions of various pion-production operators separately. We start the discussion with the longest-range leading-order pion-production operator corresponding to the Weinberg-Tomozawa (WT) diagram (Fig. 1). This LO operator is known [15] to give the most important contribution to the amplitude $M_{3 P 1}$. In Fig. 3 (left) we show the contribution to the amplitude from the WT diagram as a function of the cutoff calculated for various phenomenological NN wave functions. As expected for the longrange operator, the observables have almost no dependence on the NN interaction model. The 

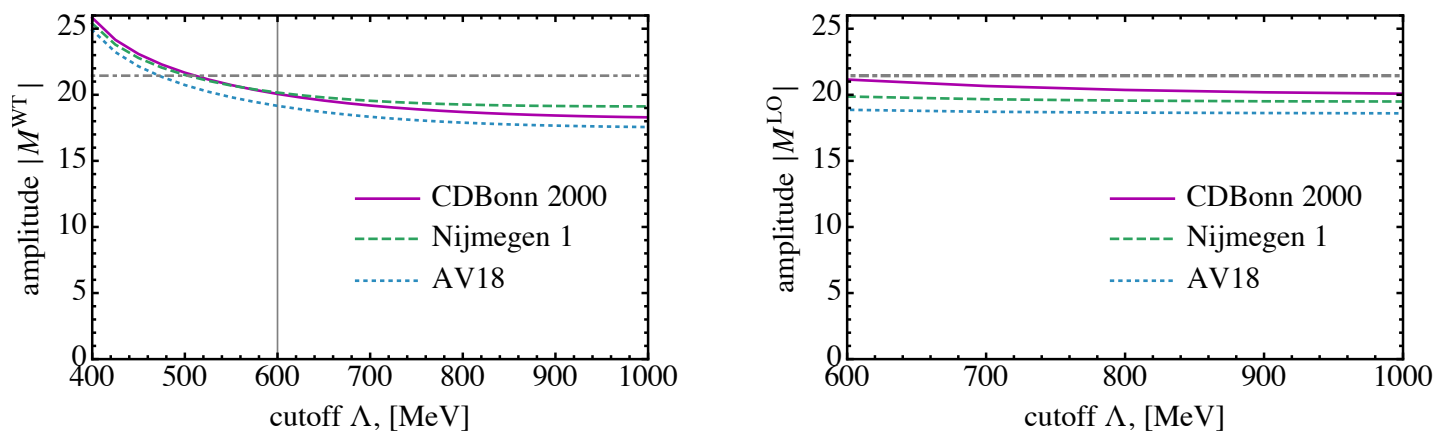

Figure 3: Contribution to the full $p p \rightarrow d \pi^{+}$amplitude $\left|M_{3 P 1}\right|$ from the LO Weinberg-Tomozawa operator (left panel) and full LO operator (right panel) as a function of cutoff calculated using various phenomenological NN wave functions. Horizontal dot-dashed line shows the experimental value of $\left|M_{3 P 1}\right|$ according to Eq. (2.6). Vertical line indicates the value of the cutoff (about $600 \mathrm{MeV}$ ) where amplitude becomes cutoff independent.

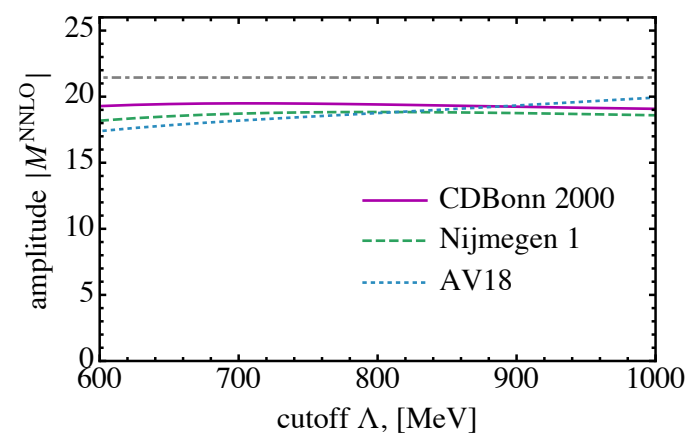

Figure 4: Contribution to the full $p p \rightarrow d \pi^{+}$amplitude $\left|M_{3 P 1}\right|$ from the full NNLO operator without contact term as a function of cutoff for several phenomenological wave functions. Horizontal dot-dashed line shows the experimental value Eq. (2.6). The difference between experimental result and displayed amplitudes corresponds to required value of the NNLO contact term.

cutoff-dependence for cutoffs $400-600 \mathrm{MeV}$ is not surprising since the typical soft scale in the pion production is about $360 \mathrm{MeV}$, and therefore for cutoffs less than about $600 \mathrm{MeV}$, the separation of scales becomes insufficient to get fully cutoff-independent result. In what follows we consider cutoffs in the range 600-1000 MeV.

The next step is to consider the full leading-order result. In addition to the WT operator (and its recoil correction), there is one more diagram at LO. This diagram is known as the direct pion production operator (Fig. 1). Since this is a single-nucleon operator, which probes NN interaction at shorter distances, it is natural to expect that the total $\mathrm{LO}$ amplitude might become more sensitive to the short-range details of the NN model. On the other hand, the contribution of direct term is known to be very small due to a destructive interference between the Born term and the contribution of the initial state interaction (see e.g. Ref. [15] where this interference was shown for CCF and CDBonn potentials). As a consequence, the direct term does not introduce any cutoff dependence, as shown in the right panel of the Fig. 3. Full LO amplitudes lie close to each other for all phenomenological potentials and reproduce reasonably well the experimental value in Eq. (2.6).

We are now in the position to consider the full NNLO amplitude based on the full NLO and 
NNLO pion-production operators, introduced in section 4. In Fig. 4 we show the pion production amplitude up-to-and-including NNLO as a function of the cutoff. Note that the contribution of the NNLO contact term (see last row in Fig. 1) is not included. Instead, the difference between the displayed NNLO result and the experimental value of $\left|M_{3 P 1}\right|$ (shown as horizontal line) illustrates the contribution of the NNLO contact term required to reproduce the data. One can see that the required contribution of NNLO contact term is very small namely about $12-18 \%$ of the LO result, and is fully consistent with the power counting. From the comparison of the LO result in Fig. 3 (right) and the NNLO result in Fig. 4, we conclude that the total contribution from all $\mathrm{NLO}^{3}$ and NNLO operators is very small, what is also in full agreement with power counting. We also observe that the results for all phenomenological NN models are in a good agreement with each other.

\section{Convolution with chiral potentials}

In this section we want to consider convolution of pion-production operators with NN wave functions based on chiral potentials, rather than phenomenological ones. This is made possible due to the recent progress in development of chiral NN potentials. In refs. $[32,33]$ the new generation of chiral NN potentials were derived up to fifth order in $M_{\pi} / \Lambda_{\chi}$. These potentials are based on the fundamental symmetries of QCD, preserve the correct analytic structure of the amplitude at low energies, and have good description of deuteron properties and NN phase shifts even for energies above pion production threshold. They are derived using a new regularization scheme, employing coordinate-space cutoffs ranging from 0.8 to $1.2 \mathrm{fm}$, which in momentum space roughly correspond to cutoffs of 500-330 MeV. Unfortunately, such cutoffs are comparable in size with threshold momentum inherent in pion production reactions. As consequence of this insufficient separation of scales, the large portion of the dynamical intermediate-range physics is transferred to the contact term which is going to be enhanced. Anyway, we have calculated the pion-production amplitude using the convolution of the operators from Sec. 4 with chiral NN wave functions and discuss our findings below.

We first consider only the longest-range LO WT pion-production operator. The result of its convolution with chiral wave functions is shown in Fig. 5 (left). As expected, the result is very similar to the one obtained with phenomenological potentials (cf. Fig. 3 left).

We next consider the full LO operator. The inclusion of the LO direct pion production operator yields the amplitude shown on the right panel of Fig. 5. We see that the contribution of the direct operator is no longer small (compared to the calculation with phenomenological NN wave functions), and it also depends on the details of $\mathrm{NN}$ potential. One can clearly see the pattern: chiral potentials with larger (in momentum space) cutoffs produce result closer to experimental data. This pattern is natural and confirms the argument given above: the smaller the momentum cut off is the more intermediate range physics is cut. The result for the chiral potential with the cutoff $R=0.8$ $\mathrm{fm}$ is already rather close to that of AV18, cf. left panels of Figs. 3 and 5. Finally, we note that the result for the full NNLO amplitude is similar to the one at LO, i.e. the inclusion of all NNLO terms does not change the pattern discussed above.

\footnotetext{
${ }^{3}$ Note that all loop diagrams at NLO were shown to cancel exactly in Ref. [15].
} 

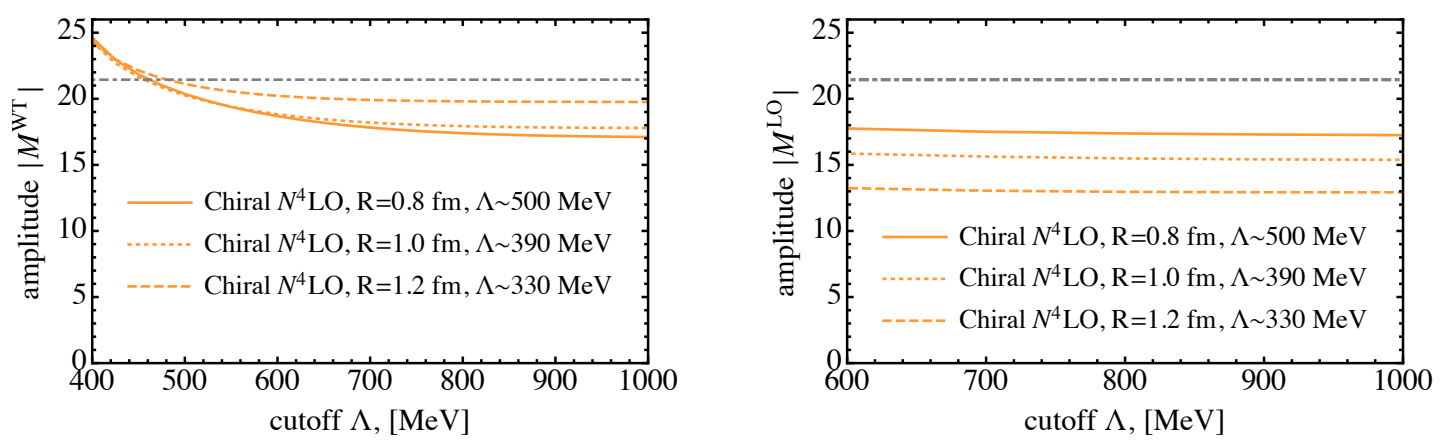

Figure 5: Contribution to the full $p p \rightarrow d \pi^{+}$amplitude $\left|M_{3 P 1}\right|$ from the Weinberg-Tomozawa operator (left) and from full LO operator (right) as a function of cutoff for chiral wave functions. Solid, dotted, and dashed orange lines correspond to results obtained using chiral wave functions constructed with coordinatespace cutoffs $1.2 \mathrm{fm}, 1.0 \mathrm{fm}$, and $0.8 \mathrm{fm}$ respectively. Horizontal gray line shows the experimental value of Eq. (2.6).

\section{Conclusion}

We have studied the s-wave pion production in $p p \rightarrow d \pi^{+}$reaction at threshold within chiral EFT. We have derived the full pion production operator up to and including NNLO in the MCS power counting which takes into account the large transferred momentum relevant for the pion production reaction. Based on the derived $N N \rightarrow N N \pi$ operators and a set of modern phenomenological and very latest chiral NN potentials, we have calculated observables in $p p \rightarrow d \pi^{+}$channel at threshold and compared them to the experimental data.

We observe that for the purpose of study $N N \rightarrow N N \pi$ reactions, the chiral potentials are generated with a cutoff, which tends to remove a part of the intermediate range physics relevant for pion production. On the other hand, assuming a resonance saturation hypothesis and modelling the short range NN interaction via heavy meson exchanges, as done in modern phenomenological calculations, yields very consistent pion production amplitudes basically independent of the NN model used. In this case the contribution of $N N \rightarrow N N \pi$ NNLO contact interaction appears to be indeed small, in agreement with the power counting estimate.

The natural next step is to study the effects of NNLO pion production operators in $p p \rightarrow p p \pi^{0}$ channel, where they are expected to play a very important role. This work is in progress.

\section{Acknowledgments}

This work was supported by the DFG (SFB/TR 16, "Subnuclear Structure of Matter"), EU HadronPhysics3 project "Study of strongly interacting matter", the European Research Council (ERC-2010-StG 259218 NuclearEFT) and the National Science Foundation Grant No. PHY1068305. This work was supported partially through GAUSTEQ (Germany and U.S. Nuclear Theory Exchange Program for QCD Studies of Hadrons and Nuclei) under contract number DESC0006758. 


\section{References}

[1] C. Hanhart, Phys. Rept. 397 (2004) 155.

[2] V. Baru, C. Hanhart and F. Myhrer, Int. J. Mod. Phys. E 23 (2014) 4, 1430004.

[3] D. S. Koltun and A. Reitan, Phys. Rev. 141 (1966) 1413.

[4] T. D. Cohen, J. L. Friar, G. A. Miller and U. van Kolck, Phys. Rev. C 53 (1996) 2661.

[5] B. Y. Park, F. Myhrer, J. R. Morones, T. Meissner and K. Kubodera, Phys. Rev. C 53 (1996) 1519.

[6] T. Sato, T. S. H. Lee, F. Myhrer and K. Kubodera, Phys. Rev. C 56 (1997) 1246.

[7] C. Hanhart and N. Kaiser, Phys. Rev. C 66 (2002) 054005.

[8] A. A. Filin et al., Phys. Rev. C 85 (2012) 054001.

[9] A. A. Filin et al., Phys. Rev. C 88 (2013) 6, 064003.

[10] G. A. Miller, A. K. Opper and E. J. Stephenson, Ann. Rev. Nucl. Part. Sci. 56 (2006) 253.

[11] A. K. Opper et al., Phys. Rev. Lett. 91 (2003) 212302.

[12] U. van Kolck, J. A. Niskanen and G. A. Miller, Phys. Lett. B 493 (2000) 65.

[13] D. R. Bolton and G. A. Miller, Phys. Rev. C 81 (2010) 014001.

[14] A. Filin et al., Phys. Lett. B 681 (2009) 423.

[15] V. Lensky et al., Eur. Phys. J. A 27 (2006) 37.

[16] T. Strauch et al., Phys. Rev. Lett. 104 (2010) 142503.

[17] T. Strauch et al., Eur. Phys. J. A 47 (2011) 88.

[18] V. Baru, E. Epelbaum, A. A. Filin, C. Hanhart, H. Krebs and F. Myhrer, arXiv:1602.07333 [nucl-th].

[19] S. Weinberg, Phys. Lett. B 295 (1992) 114.

[20] C. da Rocha, G. Miller and U. van Kolck, Phys. Rev. C 61 (2000) 034613.

[21] V. Baru et al., eConf C 070910 (2007) 128 [arXiv:0711.2748 [nucl-th]].

[22] J. A. Niskanen, Nucl. Phys. A 298 (1978) 417.

[23] M. T. Peña, H. Garcilazo, P. U. Sauer and U. Oelfke, Phys. Rev. C 45 (1992) 1487.

[24] C. Hanhart, J. Haidenbauer, O. Krehl and J. Speth, Phys. Lett. B 444 (1998) 25.

[25] C. Hanhart, J. Haidenbauer, M. Hoffmann, U.-G. Meißner and J. Speth, Phys. Lett. B 424 (1998) 8.

[26] V. Dmitrasinovic, K. Kubodera, F. Myhrer and T. Sato, Phys. Lett. B 465 (1999) 43.

[27] S.-i. Ando, T.-S. Park and D. P. Min, Phys. Lett. B 509 (2001) 253.

[28] V. Bernard, N. Kaiser and U.-G. Meißner, Eur. Phys. J. A 4 (1999) 259.

[29] R. Machleidt, Phys. Rev. C 63 (2001) 024001.

[30] V. G. J. Stoks, R. A. M. Klomp, C. P. F. Terheggen and J. J. de Swart, Phys. Rev. C 49 (1994) 2950.

[31] R. B. Wiringa, V. G. J. Stoks and R. Schiavilla, Phys. Rev. C 51 (1995) 38.

[32] E. Epelbaum, H. Krebs and U.-G. Meißner, Phys. Rev. Lett. 115 (2015) 12, 122301.

[33] E. Epelbaum, H. Krebs and U.-G. Meißner, Eur. Phys. J. A 51 (2015) 5, 53. 